\title{
Design and Preliminary Testing Plan of Electronegative Ion Thruster
}

\author{
Natalie R. Schloeder ${ }^{1}$, Thomas M. Liu ${ }^{2}$, and Mitchell L.R. Walker ${ }^{3}$ \\ Georgia Institute of Technology, Atlanta, GA 30332 \\ Kurt A. Polzin ${ }^{4}$ and John W. Dankanich ${ }^{5}$ \\ NASA George C. Marshall Space Flight Center, Huntsville, AL 35811 \\ and \\ Ane Aanesland ${ }^{6}$ \\ Laboratoire de Physique des Plasmas (LPP), CNRS-Ecole Polytechnique, 91128 Palaiseau Cedex, France
}

Electronegative ion thrusters are a new iteration of existing gridded ion thruster technology differentiated by their ability to produce and accelerate both positive and negative ions. The primary motivations for electronegative ion thruster development include the elimination of lifetime-limiting cathodes from a thruster system and the ability to generate appreciable thrust through the acceleration of both positive or negative-charged ions. Proof-of-concept testing of the PEGASES (Plasma Propulsion with Electronegative GASES) thruster demonstrated the production of positively and negatively-charged ions (argon and sulfer hexafluoride, respectively) in an RF discharge and the subsequent acceleration of each charge species through the application of a time-varying electric field to a pair of metallic grids similar to those found in gridded ion thrusters. Leveraging the knowledge gained through experiments with the PEGASES I and II prototypes, the MINT (Marshall's Ion-ioN Thruster) is being developed to provide a platform for additional electronegative thruster proof-ofconcept validation testing including direct thrust measurements. The design criteria used in designing the MINT are outlined and the planned tests that will be used to characterize the performance of the prototype are described

\section{Nomenclature}

$\begin{array}{ll}\alpha & =\text { Electronegativity } \\ d_{s} & =\text { Beamlet Diameter } \\ \gamma & =\text { Thrust Correction Factor } \\ I_{s p} & =\text { Specific Impulse } \\ J_{\text {ions }} & =\text { Sheath Thickness } \\ l_{e} & =\text { Grid Gap } \\ l_{g} & =\text { Adjusted Mass of Propellant Mixture } \\ M & =\text { Ion Mass Flow Rate } \\ \dot{m}_{i} & =\text { Ion Number Density } \\ n_{i} & =\text { Total Power Input } \\ P_{i n} & =\text { Volumetric Flow Rate } \\ Q & =\text { Electron/Ion Temperature }\end{array}$

${ }^{1}$ Graduate Student Researcher, School of Aerospace Engineering, nschloeder3@gatech.edu, Student Member AIAA

${ }^{2}$ Research Engineer, School of Aerospace Engineering, thomas.liu@ae.gatech.edu, Member AIAA

${ }^{3}$ Associate Professor, School of Aerospace Engineering, mitchell.walker@ae.gatech.edu, Associate Fellow AIAA

${ }^{4}$ Propulsion Research Engineer, Propulsion Research and Technology Applications Branch, Propulsion Systems Department, kurt.a.polzin@nasa.gov, Associate Fellow AIAA

${ }^{5}$ Program Manager, Technology Development and Transfer Office, john.dankanich@nasa.gov, Associate Fellow AIAA

${ }^{6}$ Researcher, CNRS - Ecole Polytechnique, ane.aanesland@lpp.polytechnique.fr, Member AIAA 


$\begin{array}{ll}T_{M A X} & =\text { Maximum Thrust } \\ T_{\text {opt }} & =\text { Optical Transparency } \\ t_{s} & =\text { Grid Thickness } \\ V_{a}, V_{b}, V_{S}, V_{T} & =\text { Acceleration, Beam, Screen, and Total Voltage } \\ v_{i} & =\text { Ion Velocity }\end{array}$

\section{Introduction to Electronegative Ion Thrusters}

In classical gridded ion thrusters (GITs), positively-charged ions are generated in a noble-gas fed plasma discharge and subsequently accelerated by an electric field applied between two grids to produce thrust. A separate electron source is required to neutralize the ion beam as it exits the thruster to maintain overall charge balance. ${ }^{1}$ In contrast, the electronegative thruster, pioneered by Ecole Polytechnique in 2007 with the development of the Plasma Propulsion with Electronegative GASES (PEGASES) prototype uses a propellant mixture of a noble gas (argon) with an electronegative gas (sulfur hexafluoride) to produce and accelerate both positively and negatively-charged ions. Such an approach eliminates the need for a neutralizer cathode at the thruster exit. ${ }^{2-5}$

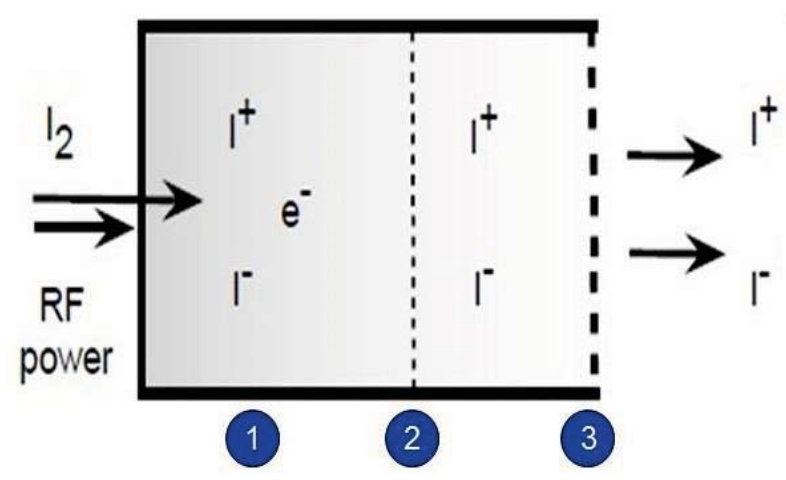

Figure 1. Three stages of an electronegative ion thruster. ${ }^{4}$

The elimination of cathodes offers several potential benefits for an electric propulsion system, most notably an increase in expected thruster lifetime and performance with a reduction in system complexity and cost. In conventional ion and Hall effect thrusters, the selection of the cathode materials, configuration, and overall structural design and assembly can be the controlling factor on thruster lifetime. In addition, the use of high-purity propellants that have greater processing costs may be necessary to prevent contamination and cathode poisoning. ${ }^{6}$ Furthermore, cathodes may require a separate propellant source and feed system. Long preheat times in hollow cathodes may reduce thruster operational flexibility. Oscillations induced through cathode and thruster plume interactions can also contribute to increased grid erosion in ion thrusters. ${ }^{7}$

In addition to eliminating the neutralizer cathode, electronegative ion thrusters may also allow for further simplification of the ion thruster electrical subsystem. In order to accelerate both species of charged particles, the screen grid of the electronegative thruster must alternate between a positive and negative bias in order to accelerate both charge species from the exit and generate thrust. Unlike conventional GITS that require a separate power supply to operate the acceleration grids, electronegative ion thrusters can use the same power supply present in the RF system due to the cyclical nature of their grid biasing scheme.

Electronegative ion thrusters are able to produce appreciable thrust using both positively and negatively-charged particles. This is a distinction from typical GITs in which the electrons are too light to contribute to thrust generation. Since both charged species in an electronegative ion thruster are heavy ions with low relative velocities and large collisional cross sections, the recombination times in the plume are shorter than typical ion-electron recombination in classical GIT plumes. Thus, electronegative thrusters reach charge neutrality in the plume more quickly mitigating potential spacecraft-plume interactions. ${ }^{4}$

Marshall's Ion-ioN Thruster (MINT) represents one alternative iteration of the electronegative thruster concept built upon the PEGASES prototypes. The MINT aims to demonstrate the feasibility of electronegative ion thrusters through direct thrust measurements while assessing the key design drivers impacting thruster operations. While significant research into the electronegative ion thruster concept has been made abroad, the MINT will be the first domestic investment in this endeavor. The scope of the MINT is to characterize the performance and system level scaling laws enabling a feasibility assessment of electronegative ion thruster technology to be made. The design criteria for building an electronegative ion thruster and the specific design of the MINT are outlined below. 

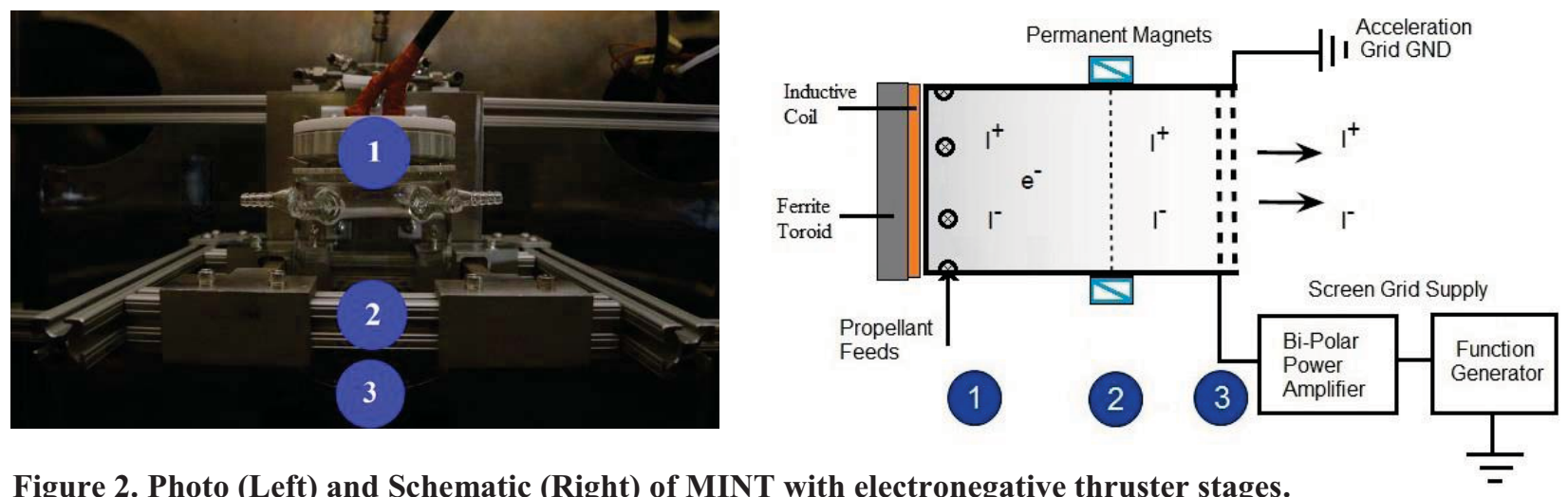

Figure 2. Photo (Left) and Schematic (Right) of MINT with electronegative thruster stages.

\section{The MINT Electronegative Ion Thruster Prototype Design}

In general, gridded electronegative ion thrusters derived from the PEGASES concept are comprised of three stages, as seen in Figure $1 .{ }^{4}$ In the first stage (source region), a plasma discharge composed of positive ions and electrons is generated and sustained via radiofrequency (RF) waves from an inductive coil. Plasma diffuses downstream into the second stage (ion-ion plasma formation region), where the electrons drop in temperature as they travel in the direction of the positive magnetic field gradient and are confined to the source region. ${ }^{8}$ The lower temperature electrons, due to their lower energy, more readily attach to neutral propellant atoms to form negative ions. These ions are more massive than the electrons and are free to pass through the applied magnetic field. Downstream of the electron filter, the resultant ion-ion plasma enters the third stage (extraction and acceleration region) in which conventional ion optics alternately extract and accelerate both ion species to generate thrust. The following subsections offer a detailed description of the MINT's design considerations.

\section{A. Plasma Source Considerations}

The main design considerations of the RF source region include the RF coupling mode, propellant injection scheme, and antenna configuration. The PEGASES I operated in the helicon mode with a single point of propellant injection for propellant and a ferromagnetic planar inductor coil. ${ }^{3,5}$

Five different RF coupling modes can be considered for an electronegative ion thruster: capacitively-coupled plasmas (CCP), very high frequency CCPs (VHCCP), inductively-coupled plasmas (ICP), ferromagnetic enhanced ICPs (FMICP), and helicon mode plasmas. ${ }^{9}$ Each RF coupling mode is distinguished by the ionization physics and the RF power required to achieve each mode. Since ICPs and FMICPs offer the most even radial distribution of ion density as compared to the other coupling modes ${ }^{10,11}$, they are the most desirable mode for gridded ion thruster operation.

In a direct comparison between ICPs and FMICPs, FMICPs offer great advantages in power transfer efficiency, defined as the ratio of power absorbed by the plasma to the power delivered to the antenna. ${ }^{9,10}$ FMICPs also typically operate at a power factor closer to unity, have the ability to produce an RF plasma at lower plasma densities, and offer better density profile control over ICPs. A ferromagnetic core enhances coupling and allows the FMICP to transfer power to the plasma in a manner that more closely resembles the power transfer in an ideal transformer. FMICPs are more easily matched to an RF source and, when properly designed, can operate more efficiently and with greater cost effectiveness over a wide range of RF power and gas pressure conditions when compared with standard ICPs. ${ }^{9}$

For the MINT source design, a ferrite toroid is used in

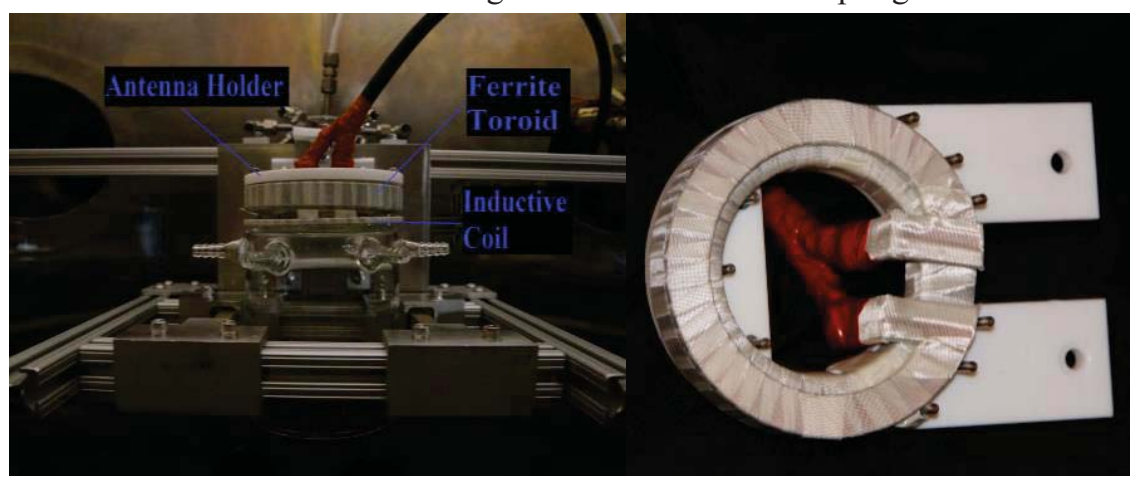

Figure 3. MINT antenna assembly from back to front: antenna mount, ferrite toroid, and inductive coil. 
conjunction with a wide, single-turn, copper antenna similar in design to a plasma processing antenna. ${ }^{9}$ The permeability of the ferrite toroid limits operation to radio frequencies less than $4 \mathrm{MHz}$ to gain the benefits of the ferrite core. This upper limit in thruster RF driving frequency helps reduce capacitive coupling and transmission line effects that can cause significant deterioration of plasma uniformity. ${ }^{9}$ Lower RF driving frequencies also permit the use of less expensive RF power sources. If the antenna is wider than the wall thickness of the discharge chamber it ensures that a significant amount of the magnetic flux from the antenna interacts with the propellant, thereby increasing ionization efficiency over thinner antennas. ${ }^{9}$ In addition,

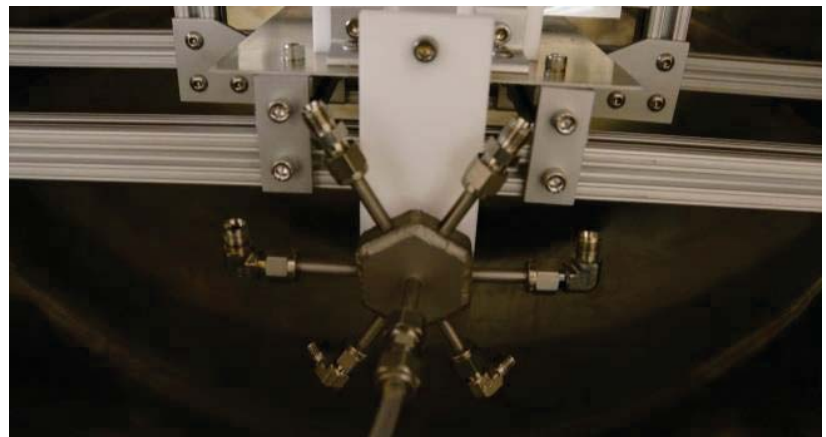

Figure 4. Gas premixer/distributor mounted near injection ports.

keeping the number of antenna turns as low as possible keeps the coil voltage to a minimum which reduces undesirable capacitive coupling. Capacitive coupling exists between the RF coil and the plasma, which causes an RF sheath to develop on the discharge chamber wall. This sheath enhances ion bombardment of the wall that increases wall erosion and plasma contamination. ${ }^{9}$

In MINT, the antenna is mounted on-axis at the extreme upstream face of the thruster. The propellant injection scheme incorporates six separate, radial gas injection ports, with equal azimuthal spacing at the upstream face of the thruster in front of the antenna assembly. This design enhances propellant density uniformity in front of the antenna to facilitate RF power coupling and propellant utilization. Computational fluid dynamics simulations were conducted with ANSYS FLUENT to ensure no unwanted flow features were introduced using this propellant injection scheme over the range of propellant mass flow rates of interest and enforcing a high vacuum pressure condition at the thruster exit. Propellant is fed to the injection ports from a gas pre-mixer/distributor that is stationed near the source region of the thruster assembly.

\section{B. Ion-Ion Plasma Formation}

Unlike typical GITs, electronegative ion thrusters generate both positive and negative ions. Generation of negative ions relies on the attachment of free electrons to neutral particles. Since electron temperatures in an RF discharge are often too high for attachment, a method of electron cooling is needed to encourage formation of negative ions. The net result is the formation of ion-ion plasma upstream of the thruster grid assembly.

Four different electronegative plasma regimes exist and are characterized by ranges of $\alpha$ (electronegativity), which is the ratio of negative ions to electrons. ${ }^{2}$ For electronegative ion thrusters, $\alpha>10^{5}$ (Regime 4) is desired because the electron density and electron flux can be considered negligible with minimal effect on the plasma sheath. ${ }^{2}$ Sheath potentials at the thruster grids can then be positive or negative allowing for accelerating fluxes of positive and negative ions to exit the thruster. Only in this regime can negative ions enter the grid sheath with the relevant Bohm

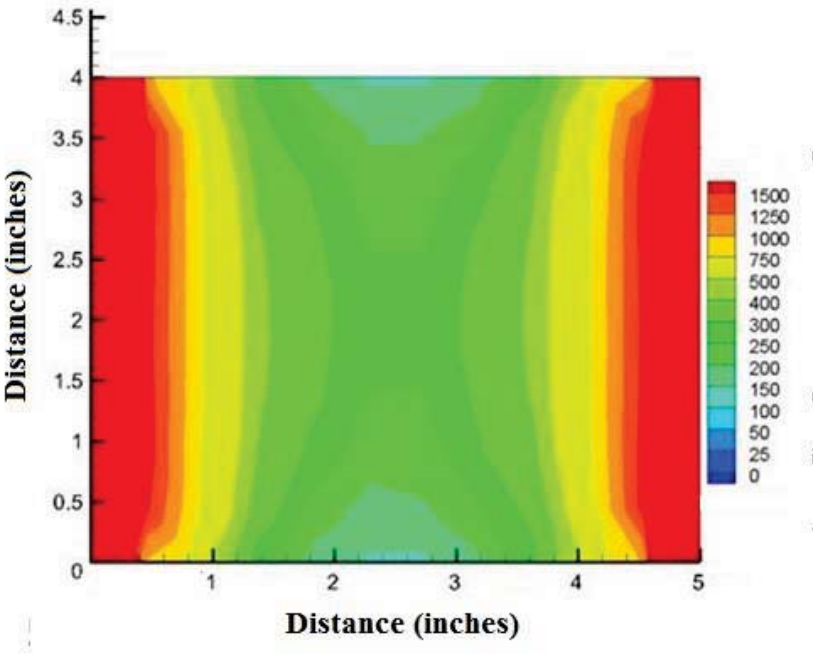

Figure 5. Slice of magnetic field topology taken along magnetic filter centerline. Plane perpendicular to bulk flow. velocity necessary to generate thrust. ${ }^{2}$

Three methods exist by which ion-ion plasma can be achieved. The first method requires pulsing the discharge that results in a decrease in electron temperature between pulses. The decrease in temperature leads to a reduction in the electron population as the negative ion formation rate increases. Pulsed operations can cause time-varying plasma density that is not conducive to thrust generation and would require additional power system complexity. ${ }^{1,12}$ 
The second method involves the use of electron beams. Unfortunately, electron beams require a separate electron source which adds system complexity and lifetime concerns, especially in the case where the electron source is a hollow cathode.

The final method is magnetic filtering, which lowers electron temperatures and traps electrons in the source region, thereby leading to an increase in negative ion production. Magnetic filtering requires low to moderate magnetic field strengths to allow ions to drift downstream perpendicular to the field while electrons are reflected back into the source. The strength of the magnetic field and its maximum position determine the formation of the ion-ion plasma region. ${ }^{3}$ Electron cooling is observed along the increasing gradient of the magnetic field after which the electron will maintain its present

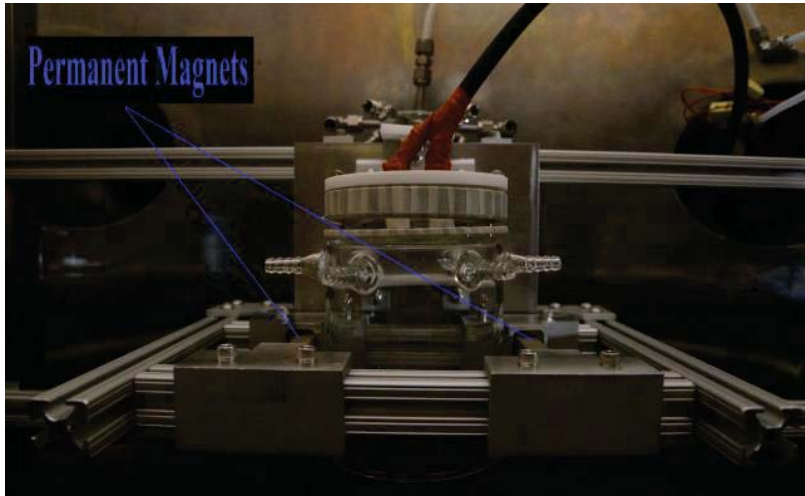

Figure 6. MINT magnetic filter made from 2 permanent neodynium magnets. temperature. The magnetic field strength can be adjusted to guarantee the best electron filtering possible. ${ }^{8}$

Magnetic filters can be constructed using either solenoid coils or permanent magnets. Considerations for the two include mass, magnetic field architecture, and ease of use. The MINT uses two neodynium magnets capable of generating a magnetic field between 100 to 250 gauss on the magnetic filter centerline. As power is increased, a higher magnet field strength may be required to maintain ion-ion plasma formation. Infolytica's MagNet was used to model the magnet separation distances necessary to achieve the desired magnetic field strengths.

\section{Extraction and Acceleration of Ions}

In typical GITs, a screen grid biased below the anode potential is used to align charged particles leaving the thruster in order to maximize thrust and limit the erosion of the downstream, negatively-biased acceleration grid. A plasma sheath forms between the two grids extracting positive ions from the plasma and accelerates them to generate thrust. Electrons are collected by an anode in the discharge region and expelled at the thruster exit through a neutralizer cathode to maintain quasineutrality of the far-field plume.

The PEGASES II considered two different methodologies for extracting and accelerating ions from an electronegative plasma. ${ }^{3,4}$ The first method relied on employing independent grid assemblies such that the positively and negatively-charged ions were separately extracted and accelerated in different spatial locations. The second method used a temporally-varying approach where a single grid assembly used an alternating voltage bias that changed from positive to negative over the course of a periodic waveform (e.g., sinusoidal, square, etc.) allowing for an alternating acceleration of positively and negatively-charged species. This approach was successfully demonstrated in references 13-16 and the MINT makes use of the same time-varying approach where bi-polar voltages are applied to ion optics.

To achieve ion extraction and acceleration, the plasma potential must follow the grid potential, and both species of charged particles must be allowed to reach the grids. ${ }^{3}$ The first condition is met by using an electrically insulating material (e.g., Pyrex) for the discharge chamber walls. The second condition is met by ensuring that an ion-ion plasma exists in the region directly upstream of the grids. The screen grid, which is in direct contact with the plasma, is biased by the application of a periodic voltage waveform, which alternates the electric field generated between the grids. This, in turn, alternatively captures and accelerates positively and negatively-charged ions downstream. Preliminary results from PEGASES II suggest that square waveforms with rise times on the order of microseconds are effective at achieving equal acceleration of positive and negative ions.

In PEGASES, grid position was found to have little influence on the formation of ion-ion plasma and the optimal location of the grids is the location of maximum $\alpha$ and highest ion density following the magnetic filter. ${ }^{3}$ As in classical ion optics design, the space charge-limited ion current density $J_{\text {ions }}$ achievable in the grid assembly is given by the Child-Langmuir law as

$$
J_{\text {ions }}=\frac{4 \varepsilon_{0}}{9}\left(\frac{2 e}{M}\right)^{\frac{1}{2}} \frac{V_{T}^{\frac{3}{2}}}{\left(l_{e}\right)^{2}}
$$

where $M$ is the adjusted mass of the propellant mixture based on the mixture ratio of argon to sulfur hexafluoride, $V_{T}$ is the voltage difference between the upstream and downstream grids, and the sheath thickness between the grids $l_{e}$. 
The sheath thickness is given as

$$
l_{e}=\sqrt{\left(l_{g}+t_{s}\right)^{2}+\frac{d_{s}^{2}}{4}}
$$

where $l_{g}$ is the grid gap, $t_{s}$ is the grid thickness, and $d_{s}$ is the beamlet diameter. ${ }^{1}$

Using the electron/ion temperature and ion number density values observed in the PEGASES, grid calculations were performed for the MINT assuming the same total volumetric flow rate of $6 \mathrm{sccm}$ and volumetric propellant mixture of $20 \%$ sulfur hexafluoride and $80 \%$ argon. The MINT grids were designed to be thin, high transparency ion optics operating near the perveance limit at a desired grid bias so as to maximize the thrust density. ${ }^{1}$ Estimated thruster performance using the MINT grid design is detailed in Table 1. The estimated thrust was calculated as

$$
T=\gamma \dot{m}_{i} v_{i}
$$

where $\gamma$ is an assumed thrust correction factor of $0.95, \dot{m}_{i}$ is the average mass flow rate of ions, and $v_{i}$ is ion velocity calculated assuming a beam voltage of $270 \mathrm{~V}$ with an assumed net-to-total voltage ratio of $0.9 .^{1}$ In calculating the ion mass flow rate, a weighted average was used taking into account the propellant mixture ratio of argon to sulfur hexafluoride. The low specific impulse (400 s) predicted for MINT is indicative of an unoptimized propellant flow rate at $6 \mathrm{sccm}$. Higher specific impulses are expected with optimized propellant flow rates during performance testing.

\begin{tabular}{clll} 
Property & \multicolumn{1}{r}{ Value } & $\underline{\text { Units }}$ & \multicolumn{1}{c}{ Description } \\
\hline $\boldsymbol{\gamma}$ & 0.958 & & Thrust Correction Factor \\
$\boldsymbol{T}_{\boldsymbol{e}, \boldsymbol{i}}$ & 0.2 & $\mathrm{eV}$ & Ion/Electron Temperature \\
$\boldsymbol{n}_{\boldsymbol{i}}$ & $2 \times 10^{11}$ & particles $/ \mathrm{cm}^{2}$ & Ion Number Density \\
$\boldsymbol{V}_{\boldsymbol{s}}$ & 300 & $\mathrm{~V}$ & Screen Grid Bias \\
$\boldsymbol{V}_{\boldsymbol{a}}$ & 0 & $\mathrm{~V}$ & Accel Grid Bias \\
$\boldsymbol{V}_{\boldsymbol{b}}$ & 270 & $\mathrm{~V}$ & Beam Voltage \\
$\boldsymbol{P}_{\boldsymbol{i n}}$ & 700 & $\mathrm{~W}$ & Total Input Power \\
$\boldsymbol{Q}$ & 6 & $\mathrm{sccm}$ & Total Volumetric Flow Rate \\
$\boldsymbol{T}_{\boldsymbol{o p t}}$ & 0.65 & & Grid Transparency \\
$\boldsymbol{J}_{\boldsymbol{i o n s}}$ & $\sim 1.2$ & $\mathrm{~mA} / \mathrm{cm}^{2}$ & Ion Current Density \\
$\boldsymbol{I}_{\boldsymbol{s} \boldsymbol{p}}$ & $\sim 400$ & $\mathrm{~s}$ & Specific Impulse \\
$\boldsymbol{T}_{\boldsymbol{M A X}}$ & $\sim 1.1$ & $\mathrm{mN}$ & Maximum Possible Thrust \\
\hline
\end{tabular}

Table 1. Calculated MINT performance estimates based on PEGASES plasma properties and anticipated operating conditions.

\section{Upcoming Performance Testing of Thruster Prototype}

The MINT is currently prepared to undergo characterization at NASA MSFC in the one of their vacuum test facilities. Testing will first be conducted in the absence of the grid assembly. Plasma properties such as plasma potential, species populations, and electron density and temperature will be measured using emissive, ExB, and Langmuir probes respectively. Operation will occur at different argon-to-sulfur hexafluoride mixture ratios, propellant flow rates, and RF power levels and frequencies to determine available RF coupling modes and plume species under each operating condition. This information will be used to determine the optimal conditions for generating an electronegative plasma at the inductively coupled plasma density we anticipate for efficient operation of the grid assembly. The above testing will also be conducted with and without the use of the magnetic filter and the ferrite core to quantify their effects on electronegative thruster operation.

Following thruster characterization without the grids, the MINT

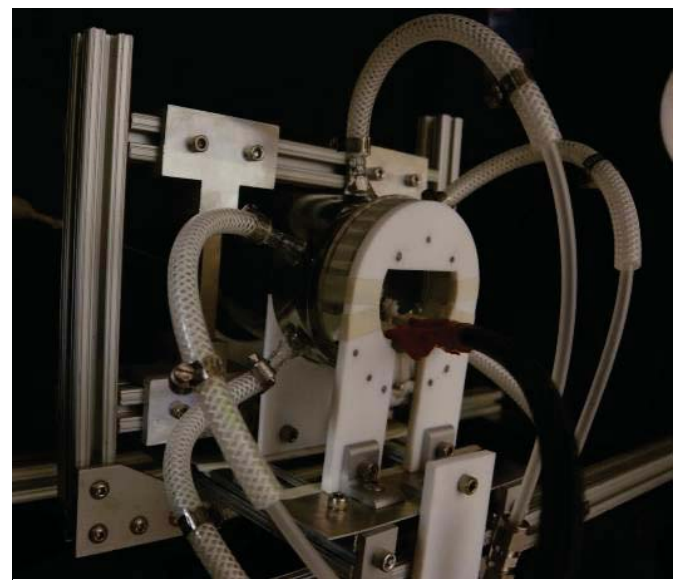

Figure 7. MINT installed in vacuum test facility at NASA MSFC. 
will be moved to a larger vacuum test facility where it will undergo direct thrust measurements with the grid assembly installed. Testing will be performed on a hanging pendulum thrust stand described in detail in Ref. 17 . A retarding potential analyzer (RPA) will be used to measure the ion energy distribution in the plume. As done in the smaller facility, ExB measurements will be performed to determine the species composition in the exhaust plume during grid operation and show the relative velocity magnitudes of both charged particle species. Emissive and Langmuir probe data will be recollected to confirm that the mode of RF coupling is unchanged during grid operation. Characterization will conclude with a system level comparison to electronegative ion thruster alternatives.

\section{Conclusion}

The MINT is an alternative design in the line of electronegative ion thrusters actively being characterized as viable alternatives to classical gridded ion thrusters. The motivation behind the development of the electronegative ion thruster includes the elimination of lifetime-limiting cathodes while generating thrust from both sets of charged species. The MINT builds upon the results of the PEGASES projects and incorporates the measured plasma values of the PEGASES II in its grid design calculations. The MINT is presently undergoing characterization at NASA MSFC through a collaboration with the Georgia Institute of Technology's HPEPL and LPP of CNRS-Ecole Polytechnique. This characterization will include direct thrust measurements, expected to be on the order of millinewtons, to validate the specific thruster design and the electronegative thruster concept in general. Following the completion of these metrics, the MINT will be used to characterize electronegative thruster operation on more exotic propellants such as iodine.

\section{Acknowledgements}

The authors would like to thank student researchers Marcellus Parker and Callen Brooks for their contributions to this project which includes simulation work and thruster set up. The authors also extend their gratitude to Michael Paul of K\&J Magnetics for all of his assistance in permanent magnet selection and modeling. Keith Chavers, Tommy Reid, Doug Galloway, Adam Martin, and Stan McDonald also contributed to the success of this project through their assistance in experimental set up and suggestions regarding data collection. Finally, the authors would like to thank the Jet Propulsion Laboratory for the use of their CEX2D grid modeling program.

\section{References}

${ }^{1}$ Goebel, Dan M., and Katz, Ira, Fundamentals of Electric Propulsion: Ion and Hall Thrusters, John Wiley \& Sons, Inc., New Jersey, 2008, Chaps. 2, 5.

${ }^{2}$ Oudini, N., Raimbault, J.-L., Chabert, P., Meige, A., \& Aanesland, A. (2013). Particle-in-cell simulation of an electronegative plasma under direct current bias studied in a large range of electronegativity. Physics of Plasmas (1994-present) 20, 043501 doi: 10.1063/1.4798501

${ }_{3}^{3}$ Aanesland1, A., Rafalskyi, D., Bredin, J., Grondein, P., Oudini2, N., \& Chabert, P. (2013, October 6-10). The PEGASES gridded ion-ion thruster performance and predictions. Presented at the 33rd International Electric Propulsion Conference, The George Washington University, Washington, D.C., USA IEPC-2013-259

${ }^{4}$ Aanesland, A., Meige, A., \& Chabert, P. (2009). Electric propulsion using ion-ion plasmas. Journal of Physics: Conference Series 162 doi:10.1088/1742-6596/162/1/012009

${ }_{5}^{5}$ Aanesland, A., Leray, G., \& Chabert, P. (2008, July 21-23). PEGASES - Plasma Propulsion with Electronegative GASES. Presented at 44th AIAA/ASME/SAE/ASEE Joint Propulsion Conference \& Exhibit, Hartford, CT, USA AIAA 2008-5198

${ }^{6}$ Polk, J. (2006, July 9-12). The Effect of Reactive Gases on Hollow Cathode Operation. Presented at the $42^{\text {nd }}$ AIAA/ASME/SAE/ASEE Joint Propulsion Conference \& Exhibit, Sacramento, California, USA AIAA 2006-5153

${ }^{7}$ Goebel, D., Jameson, K., Katz, I., \& Mikellides, I. (2007, September 17-20). Plasma Potential Behavior and Plume Mode Transitions in Hollow Cathode Discharges. Presented at $30^{\text {th }}$ International Electric Propulsion Conference, Florence, Italy IEPC-2007-277

${ }^{8}$ Aanesland, A., Bredin, J., Chabert, P., \& Godyak, V. (2012). Electron energy distribution function and plasma parameters across magnetic filters. American Institute of Physics: Appl. Phys. Lett. 100, 044102. doi: $10.1063 / 1.3680088$

${ }^{9}$ Godyak, V. (2013). TOPICAL REVIEW: Ferromagnetic enhanced inductive plasma sources. J. Phys. D: Appl. Phys. 46, 23pp. doi:10.1088/0022-3727/46/28/283001 
${ }^{10}$ Godyak, V. (2011). Electrical and plasma parameters of ICP with high coupling efficiency. Plasma Sources Sci. Technol. 20025004 (7pp) doi:10.1088/0963-0252/20/2/025004

${ }^{11}$ Franck, C., Grulke, O., \& Klinger, T. (2003) Mode transitions in helicon discharges. Phys. of Plasmas 10, 323 doi: $10.1063 / 1.1528903$

${ }^{12}$ Ashida S and Lieberman M A 1997 Japanese Journal of Applied Physics 36 854-861

${ }^{13}$ Kanakasabapathy S K and Overzet L J 1998 Plasma Sources Science Technology 7 289-297

${ }^{14}$ Kanakasabapathy S K, Overzet L J, Midha V and Economou D 2001 Applied Physics Letters 78 22-24

${ }^{15}$ Walton S G, Leonhardt D, Fernsler R F and Meger R A 2002 Applied Physics Letters 81 987-989

${ }^{16}$ Walton S G, Leonhardt D, Fernsler R F and Meger R A 2003 Applied Physics Letters 83 626-628

${ }^{17}$ Polzin, K., Markusic, T., Stanojev, B., DeHoyos, A. \& Spaun, B. (2006) Thrust stand for electric propulsion performance evaluation. Rev. Sci. Instrum. 77, 105108 (2006); doi: 10.1063/1.2357315 